\title{
Enhanced 2-h-8-day Oscillations Associated with Tropical Instability Waves
}

\author{
ZHAO JING \\ Physical Oceanography Laboratory, Ocean University of China, Qingdao, China, and Department of Oceanography, \\ Texas A\&M University, College Station, Texas \\ LIXIN Wu AND DEXING Wu \\ Physical Oceanography Laboratory, Ocean University of China, Qingdao, China
}

Bo QIU

Department of Oceanography, University of Hawai' $i$ at Mānoa, Honolulu, Hawaii

(Manuscript received 21 August 2013, in final form 17 February 2014)

\begin{abstract}
The westward South Equatorial Current (SEC) and eastward Equatorial Undercurrent (EUC) is a marginally stable current system due to the strong vertical shear. The existence of wavelike motions may locally reduce the Richardson number enough to trigger instabilities. Here, velocity measurements from the Tropical Atmosphere Ocean (TAO) array are used to examine the variability of oscillations within $0.125-12$ cycles per day (cpd). It is found that the $0.125-12$-cpd oscillations become more energetic in the presence of strong tropical instability waves (TIWs). The enhancement of shear variance is most pronounced around the EUC core $(115 \mathrm{~m})$, while prominent elevation of kinetic energy occurs around $85 \mathrm{~m}$, where the EUC shear is strongest. Particularly, the energetic $0.125-12$-cpd oscillations during strong TIW seasons do not cycle on a daily basis and are more evident during the southward phase of TIWs. The enhanced 0.125-12-cpd oscillations during strong TIW seasons can be ascribed neither to the changing background stratification nor to the vertical migration of EUC core at the corresponding time scales. Its strength is tightly correlated with the EUC shear and, to a lesser extent, the TIW shear. A partial correlation analysis suggests that the correlation to the TIW shear is mainly due to the association between EUC and TIW shear. The strength of the 0.125-12-cpd oscillations does not follow the variation of surface wind speed and wind curl, implying that they are not directly generated by surface wind forcing.
\end{abstract}

\section{Introduction}

Turbulence is an energetic, rotational, and eddying state of motion that effectively transfers its kinetic energy into heat by generating large gradients of velocity at small scales, for example, from $1 \mathrm{~mm}$ to $1 \mathrm{~cm}$ (Thorpe 2005). Energetic turbulence was found in the upper equatorial ocean (Crawford and Osborn 1981; Peters et al. 1989). The associated diapycnal transport was demonstrated to play a key role in the equatorial heat and momentum budget (Peters et al. 1988; Dillon et al. 1989; Moum et al. 2009; Moum et al. 2013). A better

Corresponding author address: Zhao Jing, Department of Oceanography, Texas A\&M University, MS 3146, College Station, TX 77840.

E-mail: jingzhao198763@sina.com knowledge of its spatial and temporal variation will improve models' representation and prediction of equatorial currents and climate variability (Pacanowski and Philander 1981; de Szoeke and Xie 2008).

Shear instability has been suggested as an important source of mixing in the stratified water column above the Equatorial Undercurrent (EUC) core (Sutherland 1996; Sun et al. 1998; Moum et al. 2011; Smyth et al. 2011; Smyth and Moum 2013; Smyth et al. 2013). The strong shear between the westward South Equatorial Current (SEC) and the eastward EUC makes the flow marginally stable (Smyth and Moum 2013). The addition of wavelike motions may locally reduce the Richardson number enough to trigger instabilities. In addition to producing turbulence, waves are also important in their own right. Using the microstructure measurements at $0^{\circ}, 140^{\circ} \mathrm{W}$, Dillon et al. (1989) reported that the estimated 
turbulent stress was not able to balance the momentum budget in the upper $30 \mathrm{~m}$. One explanation is the negligence of the momentum transport associated with the downward-radiating internal waves probably generated by perturbations of the mixed layer (Wijesekera and Dillon 1991).

In addition to large-scale circulation and small-scale turbulence, the upper equatorial ocean exhibits pronounced wavelike motions at intermediate time scales. Energetic narrowband oscillations (NBOs) have been observed in the deep cycle layer and are generally associated with bursts of turbulent mixing (Moum et al. 1992; McPhaden and Peters 1992). These oscillations are characterized by frequencies close to the local buoyancy frequency and horizontal wavelengths of 150-250 m. Its strength shows a diurnal cycle with significant enhancement during the nighttime (Moum et al. 1992; McPhaden and Peters 1992). Possible generation mechanisms include the shear instability (Smyth et al. 2013) and the perturbation of the mixed layer base (Wijesekera and Dillon 1991).

Another prominent wave motion is tropical instability waves (TIWs). TIWs are a dominant feature of seasonal variability in the equatorial Pacific Ocean (Legeckis 1977; Miller et al. 1985; Halpern et al. 1988; McPhaden 1996; Strutton et al. 2001), which can significantly affect ENSO, nutrient supply, and oceanic carbon uptake in the tropical Pacific (Murray et al. 1994; Strutton et al. 2001; Yu and Liu 2003; An 2008). In the tropical Pacific, the TIWs are visible in meanders in the equatorial sea surface temperature (SST) front (Legeckis 1977), dynamic topography (Miller et al. 1985), sea surface height (SSH) (Périgaud 1990; Farrar 2008, 2011), and ocean color (Strutton et al. 2001), as well as subsurface meridional velocity (Halpern et al. 1988) and temperature (McPhaden 1996) with periods of 15-40 days and wavelengths of $700-1600 \mathrm{~km}$. These signals are unstable modes resembling a first-meridional-mode Rossby wave centered at about $5^{\circ} \mathrm{N}$ and a surface-trapped Yanai wave on the equator (Lyman et al. 2005; Lyman 2007). The former has periods around 28-35 days and is prominent in SST and SSH (McPhaden 1996). The latter has periods around 15-23 days and produces surface-intensified meridional velocity with an amplitude of $1 \mathrm{~m} \mathrm{~s}^{-1}$ (Halpern et al. 1988; McPhaden 1996).

TIWs maintain themselves by absorbing energy from large-scale circulation through both barotropic and baroclinic instabilities (Luther and Johnson 1990). Three mechanisms may account for the generation of TIWs. The first mechanism is related to the vertical shear between the EUC and SEC (Qiao and Weisberg 1995, 1998; Luther and Johnson 1990). The second is associated with the meridional temperature gradient at the Equatorial Front (Yu et al. 1995; Luther and Johnson 1990). The third is due to the horizontal shear between the North Equatorial Countercurrent (NECC) and SEC (Philander 1978) and probably plays a minor role compared with the other two mechanisms (Proehl 1998).

The local buoyancy frequency in the upper equatorial ocean is typically $O\left(10^{-2}\right) \mathrm{rads}^{-1}$, while the frequency of TIWs is $O\left(10^{-6}\right) \mathrm{rad} \mathrm{s}^{-1}$. Therefore, there is a broad frequency gap between the NBOs and TIWs. Energetic wavelike motions with a vertical wavelength of $40 \mathrm{~m}$ and a period of 12 days were found during the cruise in April 1987 (Peters et al. 1991) and were demonstrated to play an important role in generating mixing (Peters et al. 1995). Therefore, motions within this frequency gap seem to be nontrivial, yet have not been systematically studied. In this paper, we will analyze their temporal and spatial variability using acoustic Doppler current profiler (ADCP) measurements at $0^{\circ}, 140^{\circ} \mathrm{W}$ from the Tropical Atmosphere Ocean (TAO) array. For the temporal variability, most of our studies will be based on the comparisons of their strengths during strong and weak TIW seasons. There are two motivations for choosing this composite analysis. First, it is likely that the strength of motions within the frequency gap might be related to the equatorial currents. The TIWs exhibit pronounced seasonal and interannual variability (Fig. 1) reflecting changes of equatorial currents at the corresponding time scales (Halpern et al. 1988; An 2008). Second, Moum et al. (2009) reported a unique layer of enhanced mixing immediately above the EUC core in the presence of an energetic TIW. It was suggested that the additional meridional shear produced by the TIW may nudge the flow toward instability. If shear of motions within this frequency gap were elevated at this layer during strong TIW seasons, it might also contribute to the energetic mixing.

The paper is organized as follows: Data and methodology are given in section 2. Results and analyses are presented in section 3, followed by discussions. Finally, conclusions are summarized in section 5 .

\section{Data and methods}

\section{a. Construction of TIW variability index}

We use ADCP measurements at $0^{\circ}, 140^{\circ} \mathrm{W}$ from the TAO array (McPhaden et al. 1998) to investigate TIW variability. The hourly velocity data are derived from the subsurface upward-looking ADCP with a nominal bin width and pulse length of $8 \mathrm{~m}$. These data are then linearly interpolated to a regular grid with 5-m depth intervals. The time coverage is September 1996-November 2010. 


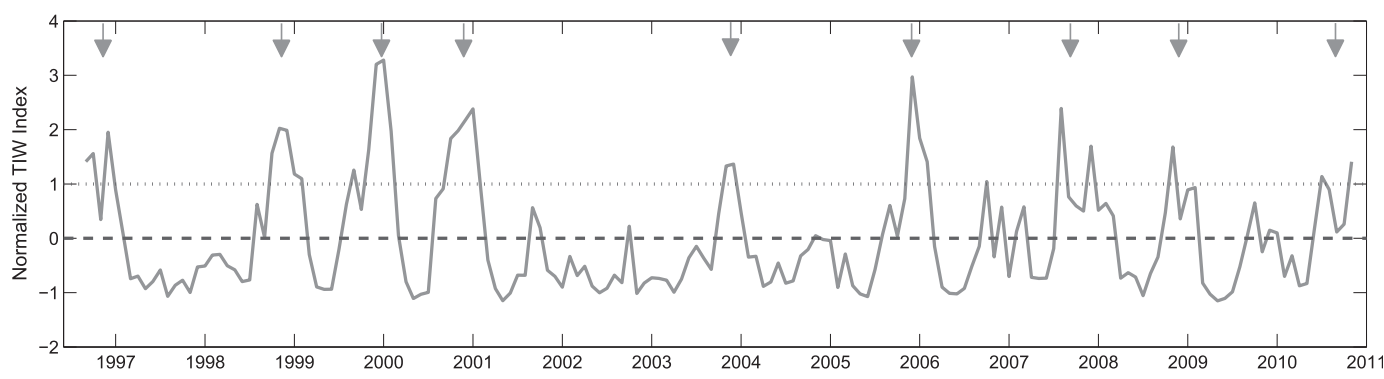

FIG. 1. Normalized TIW variability index derived from the ADCP data at $0^{\circ}, 140^{\circ} \mathrm{W}$. The gray dashed and dotted lines represent the mean and std dev of the TIW index, respectively. The strong TIW seasons (gray arrows) are defined as those with the TIW variability index one std dev larger than the mean value, while the weak TIW seasons are defined as those with the index smaller than the mean value.

The variability of TIWs is quantified following Lyman (2007). We first filter the meridional velocity data so that periods between 14 and 25 days are retained. Then a Hilbert combined empirical orthogonal function (CEOF) analysis is applied to the filtered time series to further isolate the TIW signals. The first mode exhibits great variability around the period 17.5 days and accounts for more than $80 \%$ of the total variance. We use the temporal amplitude of this first mode to indicate the variability of TIWs (Fig. 1). In this paper, the strong TIW seasons are defined as those with the TIW variability index one standard deviation larger than the mean value, while the weak TIW seasons are defined as those with the index smaller than the mean value.

\section{b. Isolating oscillations within the frequency gap}

The hourly ADCP velocity data are high-pass filtered so that periods less than 8 days are retained. The highpass-filtered signals consist of motions of $0.125-12$ cycles per day (cpd), covering a substantial portion of the frequency gap between the TIWs and NBOs. It should be noted that the cutoff period of 8 days is somewhat arbitrary. However, a moderate change of this value, that is, from 4 to 10 days, does not make any substantial impact on the following conclusions. Finally, a harmonic analysis is employed to minimize the velocity induced by tides. The harmonic analysis is applied to each 25-day bin individually rather than the whole time series in consideration of the irregularity of baroclinic tides in phase and amplitude.

\section{c. Calculating background stratification}

Gridded temperature/salinity data obtained from the Argo floats (Roemmich and Gilson 2009) are used to compute the background stratification. The monthly data span from January 2004 to December 2012 and are interpolated onto an hourly grid to match the ADCP records. The reference pressure for computing the potential density is 0 dbar.

\section{d. Calculating shear variance}

Shear variance is computed as

$$
S^{2}=\frac{(\Delta u)^{2}+(\Delta v)^{2}}{(\Delta z)^{2}},
$$

where $u$ and $v$ are the zonal and meridional components of the ADCP current, and $\Delta$ is the first-order difference operator. According to the Wentzel-KramersBrillouin (WKB) theory, waves undergo refraction in the varying background stratification. The horizontal velocity and vertical wavenumber tend to scale as $\sqrt{\bar{N} / N_{0}}$ and $\bar{N} / N_{0}$, respectively. Here $\bar{N}$ is the background buoyancy frequency and $N_{0}=10^{-2} \mathrm{~s}^{-1}$ is a reference value. To minimize the refraction effects on shear variance, the shear variance should be normalized by $\left(\bar{N} / N_{0}\right)^{3}$, henceforth referred to as the WKB-normalized shear variance. The shear variance $S_{h}^{2}$ associated with the 0.125-12-cpd oscillations is computed from (1) using the high-pass ( $<8$ day) filtered velocity. The shear variance $S_{T}^{2}$ produced by TIWs is derived from the bandpass (14-25 day) filtered meridional velocity while the EUC shear variance $S_{U}^{2}$ is computed from the lowpass ( $>25$ day) filtered zonal velocity.

\section{e. Calculating the surface wind forcing}

To examine the relation of $0.125-12$-cpd oscillations to surface wind forcing, surface wind speed and wind velocity curl are computed. The surface wind speed is derived from the measurements of the TAO mooring at $0^{\circ}, 140^{\circ} \mathrm{W}$. Its temporal resolution is $1 \mathrm{~h}$ during $1993-98$. After that, it increases to $10 \mathrm{~min}$. To be consistent with the ADCP data, the TAO wind data are hourly averaged. The TAO moorings are typically hundreds (thousands) of kilometers away from each other meridionally (zonally) and thus are not suitable to compute the relevant wind curl. The cross-calibrated multi-platform ocean surface wind vector analyses (CCMP) (Atlas et al. 2011) 

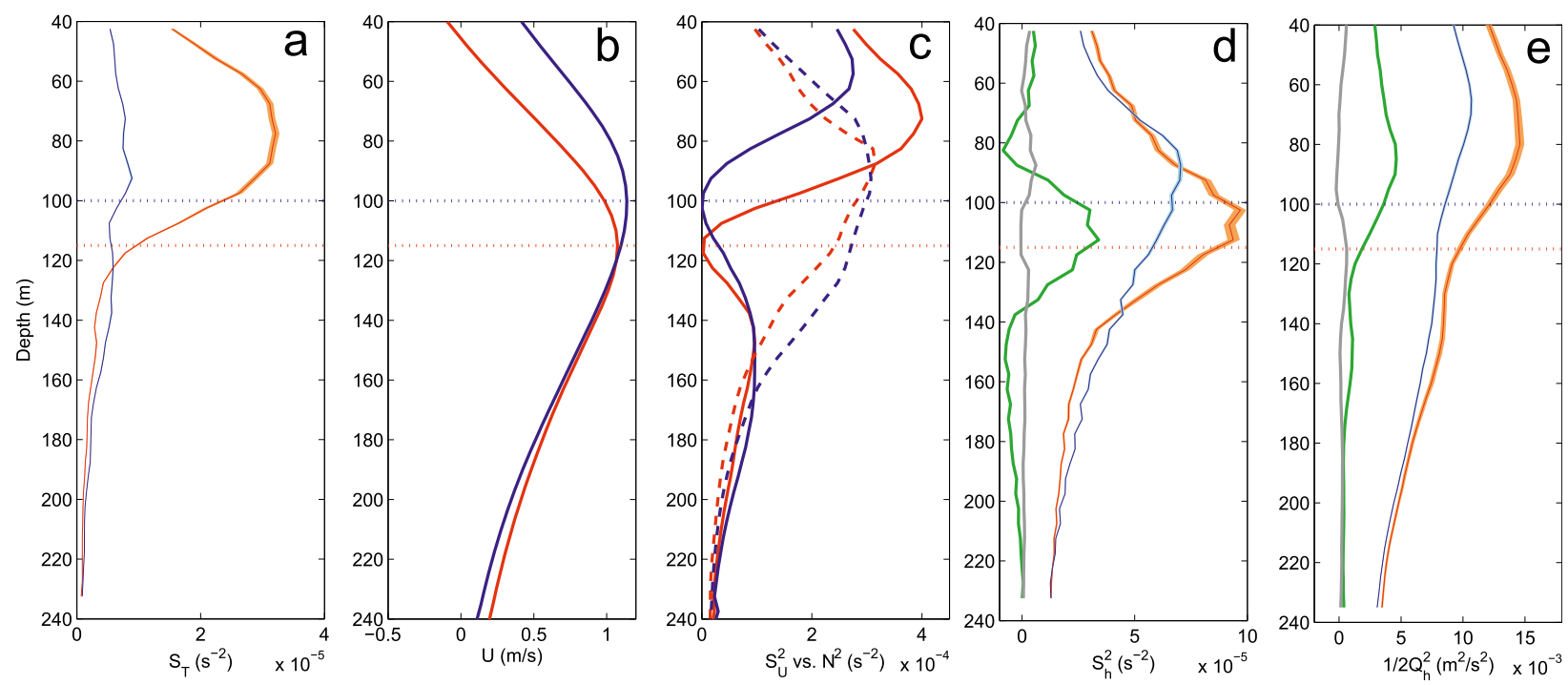

FIG. 2. Composite analyses between strong (red) and weak (blue) TIW seasons. The horizontal dotted lines mark the EUC core. (a) The TIW shear variance with the shaded regions representing the $99 \%$ confidence intervals. (b) Mean zonal flow. (c) Mean stratification (dashed) and shear variance associated with the mean zonal flow (solid). (d) Shear variance of the 0.125-12-cpd oscillations with the shaded regions representing the $99 \%$ confidence intervals. The green line denotes the difference between strong and weak TIW seasons. The gray line denotes the difference between local daytime and nighttime during strong TIW seasons. (e) As in (d), but for the kinetic energy of the $0.125-12$-cpd oscillations.

are used for this purpose. The CCMP wind data combine cross-calibrated satellite winds using a variational analysis method. It has a much higher horizontal resolution of $0.25^{\circ} \times 0.25^{\circ}$, but at the cost of a lower temporal resolution of $6 \mathrm{~h}$.

\section{Results}

\section{a. Enhanced 0.125-12-cpd oscillations during strong TIW seasons}

The TIWs exhibit pronounced seasonal and interannual variability (Fig. 1). For instance, energetic TIWs occurred during 1999-2000, while they were almost absent during 1997-98. On the equator, TIWs produce surface-intensified meridional velocity (Halpern et al. 1988; McPhaden 1996), leading to energetic meridional shear in the upper $120 \mathrm{~m}$ (Fig. 2a). The maximal speeds of the mean zonal flow between different TIW conditions are comparable to each other with both close to $1.1 \mathrm{~m} \mathrm{~s}^{-1}$ (Fig. 2b). However, the EUC core during the strong TIW seasons descends to $115 \mathrm{~m}, 15 \mathrm{~m}$ deeper than that during the weak TIW seasons. The westward SEC extends at least to $40 \mathrm{~m}$ during the strong TIW seasons, while the weak TIW seasons are associated with an eastward flow of $0.4 \mathrm{~m} \mathrm{~s}^{-1}$ at that depth (Fig. 2b). Correspondingly, shear of the mean zonal flow shows pronounced enhancement in the upper 40-100 m during the strong TIW seasons (Fig. 2c). This is consistent with the scenario where TIWs are furnished by the shear between EUC and SEC (Qiao and Weisberg 1995, 1998; Luther and Johnson 1990).

The background stratification also varies between periods of different TIW conditions. The thermocline during the strong TIW seasons becomes narrower and somewhat shallower (Fig. 2c). Particularly, the stratification in the upper 40-240 m under the strong TIW condition is weaker than that under the weak TIW condition except for $80-90 \mathrm{~m}$. The stability of the background flow is significantly reduced during the strong TIW seasons due to the enhanced EUC shear and weakened stratification.

The shear variance $S_{h}^{2}$ of the $0.125-12$-cpd oscillations is significantly elevated during the strong TIW seasons (Fig. 2d). The enhancement is most pronounced around the EUC core and extends at least to $90-130 \mathrm{~m}$. Within $90-130 \mathrm{~m}, S_{h}^{2}$ is about $38 \%$ larger during strong TIW seasons than during weak TIW seasons. To test whether the enhancement is statistically significant, a bootstrap method is applied as we have no prior information on the probability distributions of the data. The number of bootstrap samples is chosen as 1000 . Further increasing the sample number does not lead to any substantial impact on the computed statistics, but definitely requires much more computing time. The enhanced $S_{h}^{2}$ around the EUC core is a statistically robust feature during the strong TIW seasons. Its $99 \%$ confidence interval is well separated from that during the weak TIW seasons (Fig. 2d). 


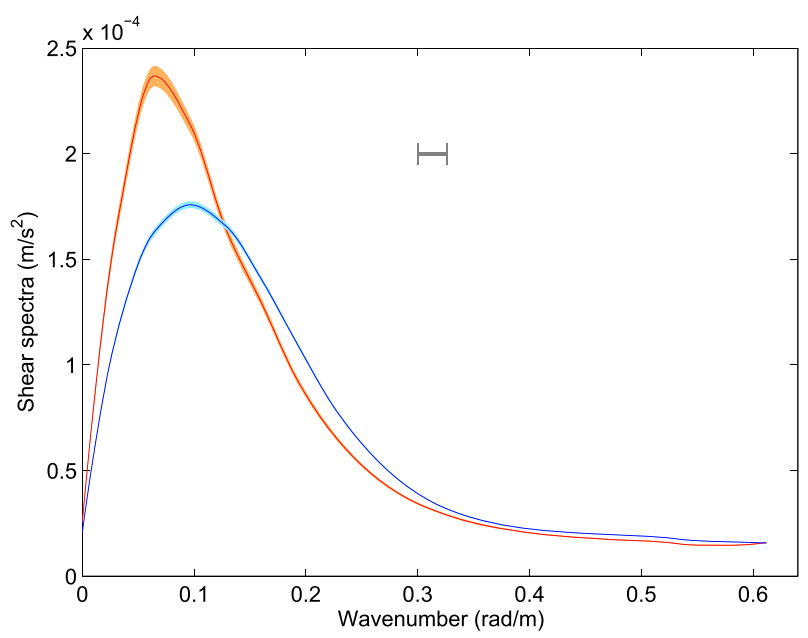

FIG. 3. Vertical wavenumber spectra of the 0.125-12-cpd shear during strong (red) and weak (blue) TIW seasons. The spectra are that of $u_{z}$ and $v_{z}$ added. The shaded regions indicate the $99 \%$ confidence intervals based on a bootstrap method. The horizontal gray bar denotes the spectral resolution of $0.026 \mathrm{rad} \mathrm{m}^{-1}$.

The energetic 0.125-12-cpd shear under the strong TIW condition is also evident in the vertical wavenumber space. Particularly, the vertical wavenumber spectra reveal that the enhancement is mainly due to the elevated shear around $0.07 \mathrm{rad} \mathrm{m}^{-1}$ (Fig. 3). The average spectral value within $0.04-0.10 \mathrm{rad} \mathrm{m}^{-1}$ is about $35 \%$ larger under the strong TIW condition than under the weak TIW condition (significant at the $<1 \%$ significance level).

The enhanced 0.125-12-cpd shear during the strong TIW seasons is unlikely to result from the aliasing of TIW signals. The depth distribution of $S_{h}^{2}$ differs from the shear variance $S_{T}^{2}$ associated with TIWs (Figs. 2a,d). The shear variance $S_{T}^{2}$ is most pronounced around $85 \mathrm{~m}$, while the enhancement of $S_{h}^{2}$ is mainly confined to 90-130 $\mathrm{m}$ and much stronger.

The EUC core typically follows the migration of the density field (Chereskin et al. 1986; Toole et al. 1987), while cross-isopycnal movements were also observed (Peters et al. 1991). Such movements may contaminate frequency spectra of velocity (Chereskin et al. 1986) and shear. The EUC shear is weakest around its core. When the EUC core migrates upward or downward, the position with minimal background shear variance moves correspondingly. In this case, the background shear variance around $115 \mathrm{~m}$ is increased with the enhancement generally proportional to the migrating distance. We compare the $0.125-12$-cpd migrating displacement under strong and weak TIW conditions. Their probability density functions (PDFs) do not exhibit any evident difference between different conditions (Fig. 4a). Particularly, the root-mean-square migrating displacement $d_{\mathrm{rms}}$ is around $7.3 \mathrm{~m}$ during both strong and weak TIW seasons. Furthermore, we estimate the contribution of EUC migration to $S_{h}^{2}$ by assuming that the vertical migration of the EUC core can be treated as a vertical translation with its shape unchanged. PDFs of the simulated shear variance are very different from the observed ones (Figs. 4b,c). Under the strong TIW condition, the mean (median) value of the simulated shear variance around $115 \mathrm{~m}$ is only about $3.2(0.68) \times$ $10^{-5} \mathrm{~s}^{-2}$ (Fig. 4b), much smaller than the observed value 9.4(4.6) $\times 10^{-5} \mathrm{~s}^{-2}$ (Fig. 4b). Similar is the case under the weak TIW condition (Fig. 4c). Moreover, the vertical wavenumber spectra suggest that $S_{h}^{2}$ is dominated by wavelengths around $80-90 \mathrm{~m}$ (Fig. 3). If the $0.125-$ 12-cpd shear was basically an artifact due to the EUC migration, its vertical scale would be related to that of
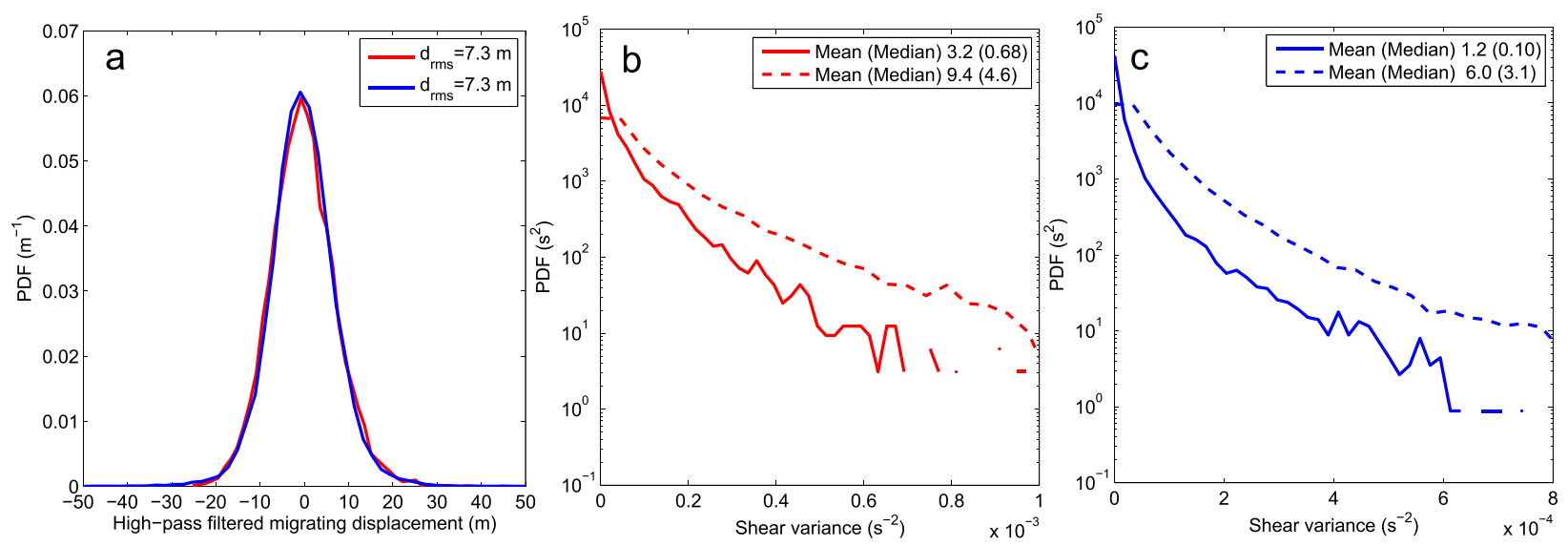

FIG. 4. (a) PDFs of the high-pass-filtered EUC migration displacement during strong (red) and weak (blue) TIW seasons. The legend shows the std dev of the migration displacement. (b) PDFs of $S_{h}^{2}$ (dashed) and the simulated shear variance (solid) during strong TIW seasons. The legend shows the mean and median values. (c) As in (b), but for the weak TIW seasons. 


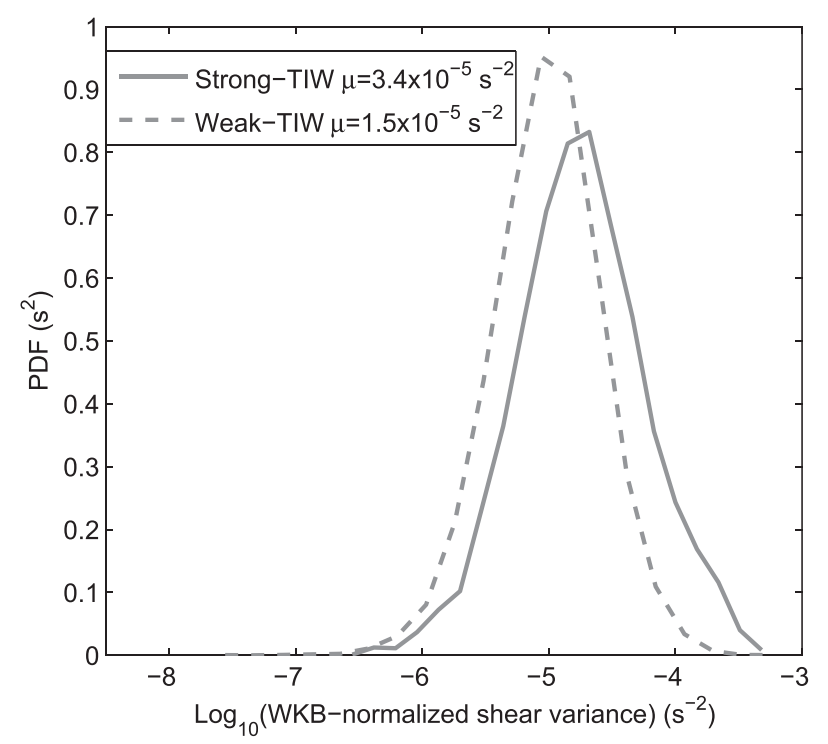

FIG. 5. PDFs of the WKB-normalized $S_{h}^{2}$ within $100-115 \mathrm{~m}$ during strong and weak TIW seasons. The legend shows the mean values. Note that a logarithm scale is used for the $x$ axis.

isopycnal migration. Previous observations at this site indicated that the isopycnal migration was in phase within 50-150 $\mathrm{m}$ (Toole et al. 1987), implying a wavelength longer than $100 \mathrm{~m}$. Therefore, the energetic $S_{h}^{2}$ during the strong TIW seasons cannot be simply ascribed to the migration of the EUC core.

The elevated 0.125-12-cpd shear during the strong TIW seasons does not result from the seasonal variation of the background stratification (Fig. 5) either. The enhancement of the WKB-normalized shear variance around the EUC core is even more evident (significant at the $<1 \%$ significance level). This is probably due to the shoaling thermocline during the strong TIW seasons (Fig. 2c).

The kinetic energy $(1 / 2) Q_{h}^{2}$ of the $0.125-12$-cpd oscillations is also elevated in the presence of energetic TIWs (Fig. 2e). Unlike $S_{h}^{2}$, the enhancement of (1/2) $Q_{h}^{2}$ is most evident around $85 \mathrm{~m}$ and extends to $40-120 \mathrm{~m}$. Reasons for the different vertical structures between $S_{h}^{2}$ and $(1 / 2) Q_{h}^{2}$ remain unknown and deserve investigation in future studies. Finally, it should be noted that both $(1 / 2) Q_{h}^{2}$ and $S_{h}^{2}$ do not cycle on a daily basis (Figs. 2d,e), suggesting that the 0.125-12-cpd oscillations are not related to the deep cycle layer mixing (Moum et al. 1992; McPhaden and Peters 1992; Lien et al. 1995).

\section{b. Correlation of the 0.125-12-cpd oscillations to winds, TIWs, and EUC}

As is demonstrated above, the $0.125-12$-cpd oscillations are significantly enhanced in the presence of strong TIWs, but it does not necessarily mean that they
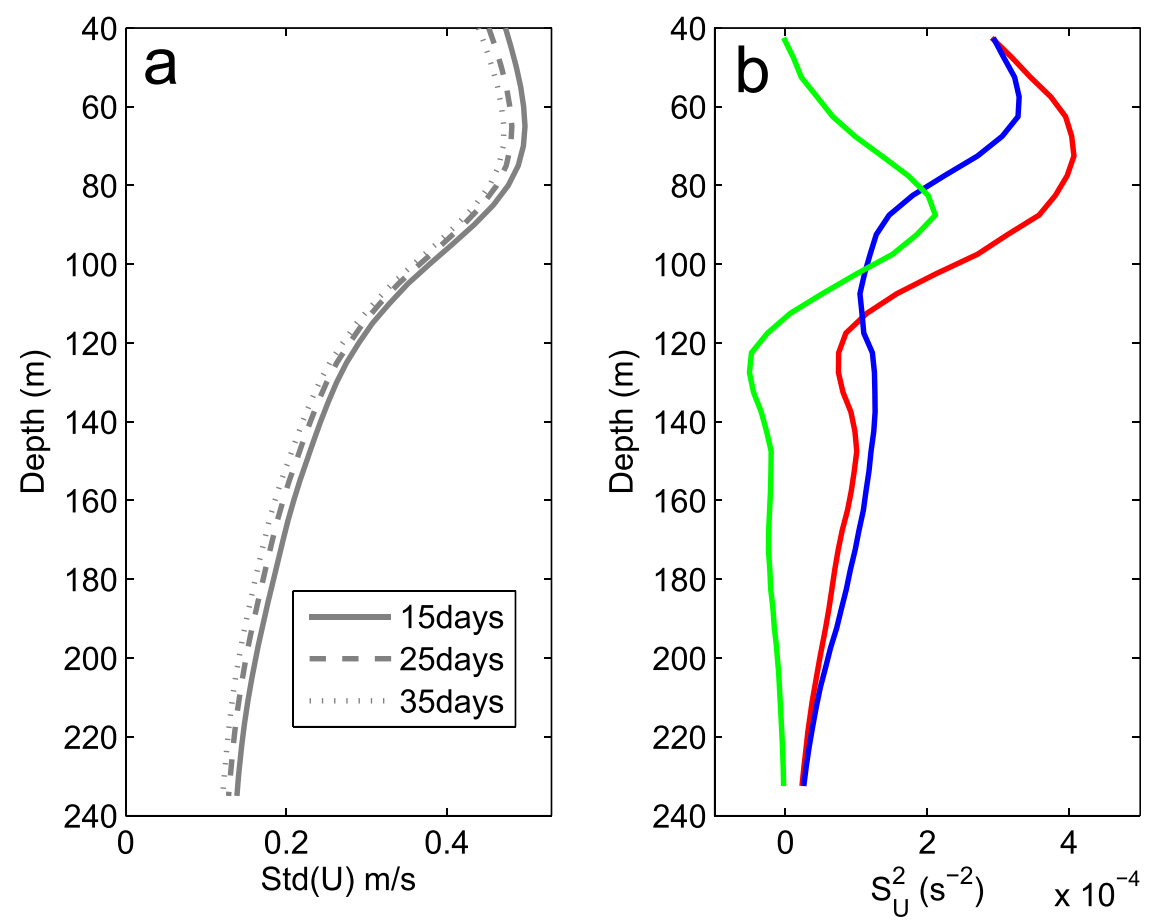

FIG. 6. (a) Std dev of the low-pass-filtered zonal velocity using different cutoff periods; (b) the EUC shear variance during strong (red) and weak (blue) TIW seasons and their difference (green). 

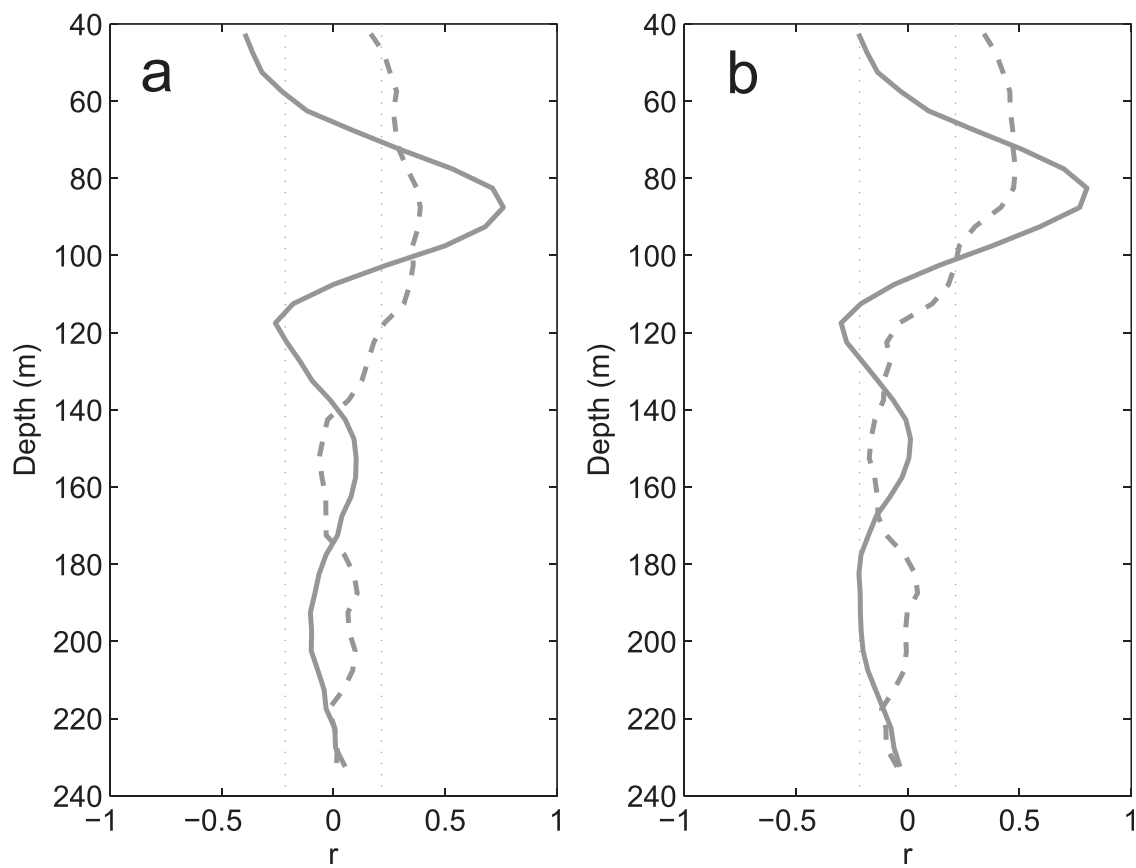

FIG. 7. (a) Correlation of $I_{S}$ with $S_{U}^{2}$ (solid) with $S_{T}^{2}$ (dashed) at different depths. The vertical dotted lines denote the $1 \%$ significance level. (b) As in (a), but for $I_{Q}$.

are directly related to TIWs, as the EUC and surface winds also vary during strong and weak TIW seasons. In this section, we will evaluate the relevance of these candidates basing on correlation analyses.

The EUC is isolated by low-pass filtering the zonal velocity with a cutoff period of 25 days. In this case, influences of internal waves and TIWs are probably removed. However, using longer or shorter cutoff periods does not make any substantial impact (Fig. 6a). This is mainly because EUC is dominated by the variations at seasonal and longer time scales.

The shear associated with EUC $S_{U}$ is significantly elevated during the strong TIW seasons (Fig. 6b). This is not surprising as the shear between EUC and SEC is in favor of generating TIWs (Qiao and Weisberg 1995, 1998; Luther and Johnson 1990). Similar to $S_{T}$ (Fig. 2b), the enhancement of $S_{U}$ is also most pronounced around $85 \mathrm{~m}$ (Fig. 6b). However, its magnitude is much larger compared with $S_{T}$. Particularly, $S_{U}^{2}$ around $85 \mathrm{~m}$ is about $4.0 \times 10^{-4} \mathrm{~s}^{-2}$ during the strong TIW seasons, an order of magnitude larger than $S_{T}^{2}$.

We implement a linear correlation analysis between the strength of $0.125-12$-cpd oscillations and $S_{U}^{2}$ and $S_{T}^{2}$ at different depths. Here, two measurements are used to indicate the strength of $0.125-12$-cpd oscillations. The first $I_{S}$ is $S_{h}^{2}$ averaged within $105-115 \mathrm{~m}$, while the second $I_{Q}$ is $(1 / 2) Q_{h}^{2}$ averaged within $75-95 \mathrm{~m}$. As is demonstrated in Fig. 7, these two measurements give very similar results. Therefore, only the results based on $I_{S}$ are documented henceforth. Before computing the correlation coefficients, we divide the data into 25-day bins and calculate the averages for each bin. There are two advantages for using the bin-averaged values. First, the random noise is significantly reduced, making the computed correlation coefficients more robust. Second, the $0.125-12$-cpd oscillations might be generated by shear instabilities of the background flow. Time is needed for energy to transfer from the background flow to instability waves. If this were the case, the background shear might be not simultaneously correlated with the strength of $0.125-12$-cpd oscillations. However, the time lag effect is almost negligible for the bin-averaged values.

The measurement $I_{S}$ is tightly correlated with $S_{U}^{2}$ around $85 \mathrm{~m}$ (Fig. 7a), where it is strongest during the strong TIW seasons (Fig. 6b). The correlation coefficient reaches up to 0.76 (significant at the $<1 \%$ significance

TABLE 1. Correlation $r$ and partial correlation $r_{p}$ of $I_{S}\left(I_{Q}\right)$ with $S_{U}^{2}$ and $S_{T}^{2}$ at $85 \mathrm{~m}$. The $p$ value represents the significance level.

\begin{tabular}{lcc}
\hline & $S_{U}^{2}$ & $S_{T}^{2}$ \\
\hline$r$ & $0.76(0.79)$ & $0.39(0.42)$ \\
$p$ value & $0(0)$ & $0(0)$ \\
$r_{p}$ & $0.71(0.72)$ & $0.11(0.15)$ \\
$p$ value & $0(0)$ & $0.19(0.06)$ \\
\hline
\end{tabular}



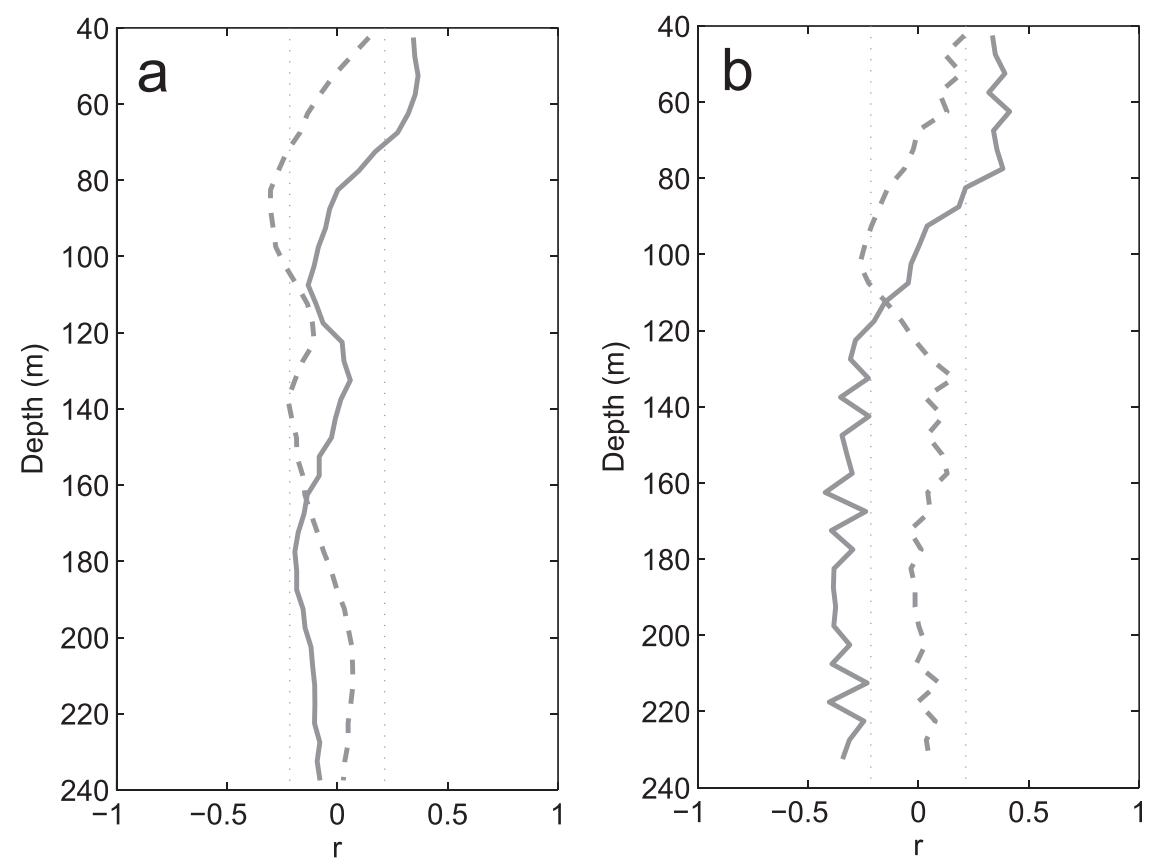

FIG. 8. Correlation of $S_{h}^{2}$ (solid) and (1/2) $Q_{h}^{2}$ (dashed) with the magnitude of the (a) $0.125-12$-cpd wind velocity and (b) $0.125-12$-cpd wind velocity curl.

level). Furthermore, there is also significant correlation between $I_{S}$ and $S_{T}^{2}$, although the correlation coefficient is much smaller (Fig. 7a). At this stage, it appears that both the EUC and TIW shear may contribute to the energetic 0.125-12-cpd oscillations during the strong TIW seasons. As $S_{T}^{2}$ and $S_{U}^{2}$ are positively correlated with each other, a partial correlation analysis is used to further isolate their contributions. In probability theory and statistics, partial correlation measures the correlation between two random variables after removing the effect of other variables (Guilford and Fruchter 1973). It helps spot spurious correlations and is suitable for our purpose here.

Table 1 lists the correlation coefficients $r$ and partial correlation coefficients $r_{p}$ between $I_{S}$ and $S_{U}^{2}\left(S_{T}^{2}\right)$ at $85 \mathrm{~m}$. Values at $85 \mathrm{~m}$ are used because it is the depth at which $S_{U}^{2}$ and $S_{T}^{2}$ exhibit the highest correlation with $I_{S}$ (Fig. 7). While $r_{p}$ is comparable to $r$ for $S_{U}^{2}, r_{p}$ is close to zero and statistically insignificant for $S_{T}^{2}$. Therefore, the correlation of $I_{S}$ with $S_{T}^{2}$ does not necessarily mean causation. Instead, it might be an artifact resulting from its correlation to $S_{U}^{2}$.

Finally, let us examine the relation of the $0.125-12$-cpd oscillations to the surface winds. The correlation coefficient between the speed of 0.125-12-cpd winds and $S_{h}^{2}\left[(1 / 2) Q_{h}^{2}\right]$ is less than 0.4 throughout the water column (Fig. 8a). Similar is the case for the magnitude of the $0.125-12$-cpd wind velocity curl (Fig. 8b). Therefore, the bulk of the variance of the $0.125-12-\mathrm{cpd}$ oscillations cannot be explained by surface wind forcing. The surface winds are unlikely to play an important role here.

\section{Discussions}

\section{a. Influences of the 0.125-12-cpd oscillations on diapycnal mixing}

Shear instability has been suggested as an important source of mixing in the stratified water column above the EUC core. During the strong TIW seasons, the shear associated with the EUC and TIWs is significantly enhanced. The addition of the energetic $0.125-12$-cpd oscillations may nudge the flow toward instability. Moum et al. (2009) reported a unique 20-40-m-thick layer of strong mixing immediately above the EUC core in the presence of an energetic TIW. As is shown in this paper, the shear associated with the $0.125-12$-cpd oscillations exhibits pronounced enhancement within 90-130 $\mathrm{m}$ during the strong TIW seasons. In addition to the vigorous EUC and TIWs, it might be possible that the energetic $0.125-12$-cpd oscillations also contribute to the mixing. We note that shear of the $0.125-12$-cpd oscillations and mixing immediately above the EUC core share two similar features. First, both do not cycle on a daily basis (Fig. 2d; Moum et al. 2009). Second, both show enhancement during the southward phase of TIWs (Fig. 9; Inoue et al. 2012). Nevertheless, 


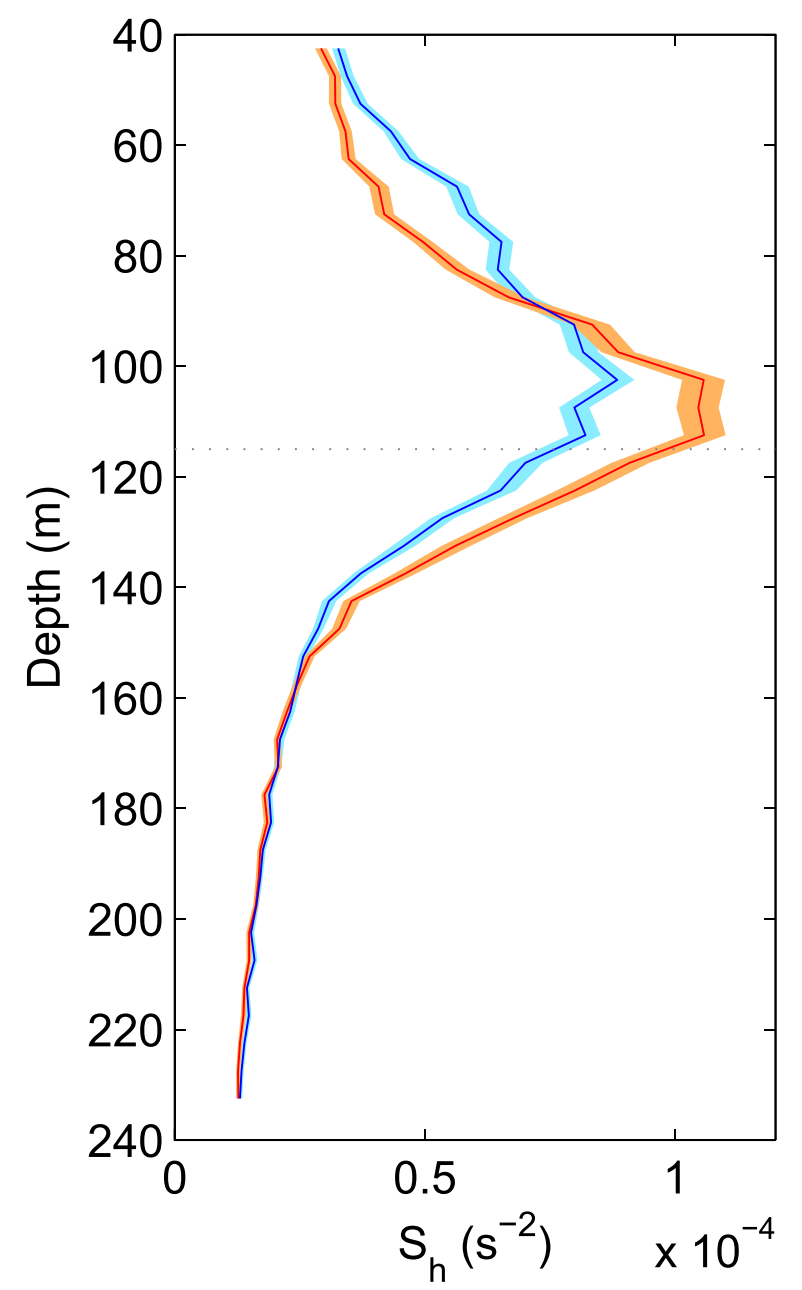

FIG. 9. The $S_{h}^{2}$ under the southward (red) and northward (blue) phase of TIWs during the strong TIW seasons. The shaded regions represent the $99 \%$ confidence intervals. The gray dotted line marks the EUC core.

a dynamical link between the mixing and $0.125-12$-cpd oscillations cannot be justified due to the limited data.

\section{b. Generation mechanisms for the 0.125-12-cpd oscillations}

As is demonstrated above, the strength of the 0.12512-cpd oscillations is tightly correlated with the EUC shear, implying that these energetic oscillations might be generated by the shear instability of EUC. Furthermore, the observed vertical structure of the $0.125-12$-cpd oscillations is not inconsistent with shear instability waves. According to the linear stability analysis (Pham et al. 2012; Smyth et al. 2013), the perturbation kinetic energy should be concentrated around the critical level typically associated with strong background shear. But this is not necessarily the case for the perturbation shear variance due to the complicated structure of the unstable modes (Sun et al. 1998; Pham et al. 2012; Smyth et al. 2013). These patterns are similar to the observations here. In a word, existing observational evidence does not rule out the shear instability as a generation mechanism.

\section{Summary}

Velocity measurements from the TAO array are used to examine the variability of $0.125-12$-cpd oscillations during strong and weak TIW seasons. In the presence of strong TIWs, the 0.125-12-cpd oscillations become more energetic. The enhancement of $S_{h}^{2}$ is most pronounced around the EUC core $(115 \mathrm{~m})$, while prominent elevation of $(1 / 2) Q_{h}^{2}$ occurs around $85 \mathrm{~m}$, where the EUC shear is strongest. Particularly, the energetic $0.125-12$-cpd oscillations during strong TIW seasons do not cycle on a daily basis and are more evident during the southward phase of TIWs.

The variability of the $0.125-12$-cpd oscillations between strong and weak TIW seasons is not due to the changing background stratification, and neither can it be ascribed to the vertical migration of EUC core at the corresponding time scales. The strength of the $0.125-$ 12-cpd oscillations is tightly correlated with the EUC shear and, to a lesser extent, the TIW shear. However, its correlation to the TIW shear is mainly due to the association between EUC and TIW shear according to the partial correlation analysis. The strength of the 0.125-12-cpd oscillations does not follow the variation of surface wind forcing at the corresponding time scales, suggesting that surface winds do not play an important role.

Acknowledgments. This work is supported by China National Natural Science Foundation (NSFC) Key Project (41130859) and NSFC Creative Group Project (40921004). Jing is partially supported by the Chinese Scholarship Council. We thank the NOAA's TAO Project office for the equatorial mooring data through online access (www.pmel.noaa.gov/tao/) and PO.DAAC for the CCMP data (ftp://podaac-ftp.jpl.nasa.gov/allData/ $\mathrm{ccmp} /$ ). Dean Roemmich and John Gilson are appreciated for providing the gridded temperature/salinity data online (www.argo.ucsd.edu/Marine_Atlas.html).

\section{REFERENCES}

An, S., 2008: Interannual variations of tropical instability waves and ENSO. J. Climate, 21, 3680-3683, doi:10.1175/2008JCLI1701.1. Atlas, R., R. N. Hoffman, J. Ardizzone, S. M. Leidner, J. C. Jusem, D. K. Smith, and D. Gombos, 2011: A cross-calibrated, multiplatform ocean surface wind velocity product for meteorological and oceanographic applications. Bull. Amer. Meteor. Soc., 92, 157-174, doi:10.1175/2010BAMS2946.1. 
Chereskin, T. K., J. N. Moum, P. J. Stabeno, D. R. Caldwell, C. A. Paulson, L. A. Regier, and D. Halpern, 1986: Fine-scale variability at $140^{\circ} \mathrm{W}$ in the equatorial Pacific. J. Geophys. Res., 91, 12 887-12 897, doi:10.1029/JC091iC11p12887.

Crawford, W. R., and T. R. Osborn, 1981: Control of equatorial ocean currents by turbulent dissipation. Science, 212, 539-540, doi:10.1126/science.212.4494.539.

de Szoeke, S. P., and S.-P. Xie, 2008: The tropical eastern Pacific seasonal cycle: Assessment of errors and mechanisms in IPCC AR4 coupled ocean-atmosphere general circulation models. J. Climate, 21, 2573-2590, doi:10.1175/2007JCLI1975.1.

Dillon, T. M., J. N. Moum, T. K. Chereskin, and D. R. Caldwell, 1989: Zonal momentum balance at the equator. J. Phys. Oceanogr., 19, 561-570, doi:10.1175/1520-0485(1989)019<0561: ZMBATE $>2.0 . \mathrm{CO}$; .

Farrar, J. T., 2008: Observations of the dispersion characteristics and meridional sea level structure of equatorial waves in the Pacific Ocean. J. Phys. Oceanogr., 38, 1669-1689, doi:10.1175/ 2007JPO3890.1.

_ - 2011: Barotropic Rossby waves radiating from tropical instability waves in the Pacific Ocean. J. Phys. Oceanogr., 41, 1160-1181, doi:10.1175/2011JPO4547.1.

Guilford, J. P., and B. Fruchter, 1973: Fundamental Statistics in Psychology and Education. McGraw-Hill, 576 pp.

Halpern, D., R. A. Knox, and D. S. Luther, 1988: Observations of 20-day meridional current oscillations in the upper ocean along the Pacific equator. J. Phys. Oceanogr., 18, 1514-1534, doi:10.1175/1520-0485(1988)018<1514:OODPMC >2.0.CO;2.

Inoue, R., R.-C. Lien, and J. N. Moum, 2012: Modulation of equatorial turbulence by a tropical instability wave. $J$. Geophys. Res., 117, C10009, doi:10.1029/2011JC007767.

Legeckis, R., 1977: Long waves in the eastern equatorial Pacific Ocean: A view from a geostationary satellite. Science, 197, 1179-1181, doi:10.1126/science.197.4309.1179.

Lien, R. C., D. R. Caldwell, M. C. Gregg, and J. N. Moum, 1995: Turbulence variability at the equator in the central Pacific at the beginning of the 1991-1993 El Niño. J. Geophys. Res., 100, 6881-6898, doi:10.1029/94JC03312.

Luther, D. S., and E. S. Johnson, 1990: Eddy energetics in the upper equatorial Pacific during Hawaii-to-Tahiti Shuttle experiment. J. Phys. Oceanogr., 20, 913-944, doi:10.1175/ 1520-0485(1990)020<0913:EEITUE > 2.0.CO;2.

Lyman, J. M., 2007: Distinct 17- and 33-day tropical instability waves in subsurface observations. J. Phys. Oceanogr., 37, 855-872, doi:10.1175/JPO3023.1.

- D. B. Chelton, R. A. deSzoeke, and R. M. Samelson, 2005: Tropical instability waves as a resonance between equatorial Rossby waves. J. Phys. Oceanogr., 35, 232-254, doi:10.1175/ JPO-2668.1.

McPhaden, M. J., 1996: Monthly period oscillations in the Pacific North Equatorial Countercurrent. J. Geophys. Res., 101, 6337-6359, doi:10.1029/95JC03620.

__ , and H. Peters, 1992: Diurnal cycle of internal wave variability in the equatorial Pacific Ocean: Results from moored observations. J. Phys. Oceanogr., 22, 1317-1329, doi:10.1175/ 1520-0485(1992)022<1317:DCOIWV>2.0.CO;2.

_- and Coauthors, 1998: The tropical ocean-global atmosphere observing system: A decade of progress. J. Geophys. Res., 103, 14169-14240, doi:10.1029/97JC02906.

Miller, L., D. R. Watts, and M. Wimbush, 1985: Oscillations of dynamic topography in the eastern equatorial Pacific. J. Phys. Oceanogr., 15, 1759-1770, doi:10.1175/1520-0485(1985)015<1759: OODTIT $>2.0 . \mathrm{CO} ;$.
Moum, J. N., D. Hebert, C. A. Paulson, and D. R. Caldwell, 1992: Turbulence and internal waves at the equator. Part I: Statistics from towed thermistors and a microstructure profiler. J. Phys. Oceanogr., 22, 1330-1345, doi:10.1175/1520-0485(1992)022<1330: TAIWAT $>2.0 . \mathrm{CO} ; 2$.

, R.-C. Lien, A. Perlin, J. D. Nash, M. C. Gregg, and P. J. Wiles, 2009: Sea surface cooling at the equator by subsurface mixing in tropical instability waves. Nat. Geosci., 2, 761-765, doi:10.1038/ ngeo657.

, J. D. Nash, and W. D. Smyth, 2011: Narrowband oscillations in the upper equatorial ocean. Part I: Interpretation as shear instabilities. J. Phys. Oceanogr., 41, 397-411, doi:10.1175/2010JPO4450.1.

_- A. Perlin, J. D. Nash, and M. J. McPhaden, 2013: Seasonal sea surface cooling in the equatorial Pacific cold tongue controlled by ocean mixing. Nature, 500, 64-67, doi:10.1038/nature12363.

Murray, J., R. T. Barber, M. Roman, M. Bacon, and R. Feely, 1994: Physical and biological controls on carbon cycling in the equatorial Pacific. Science, 266, 58-65, doi:10.1126/ science.266.5182.58.

Pacanowski, R. C., and S. G. H. Philander, 1981: Parameterization of vertical mixing in numerical models of tropical oceans. J. Phys. Oceanogr., 11, 1443-1451, doi:10.1175/1520-0485(1981)011<1443: POVMIN $>2.0 . \mathrm{CO} ; 2$.

Périgaud, C., 1990: Sea level oscillations observed with Geosat along the two shear fronts of the Pacific North Equatorial Countercurrent. J. Geophys. Res., 95, 7239-7248, doi:10.1029/ JC095iC05p07239.

Peters, H., M. C. Gregg, and J. M. Toole, 1988: On the parameterization of equatorial turbulence. J. Geophys. Res., 93, 11991218, doi:10.1029/JC093iC02p01199.

,$- \ldots$, and — 1989: Meridional variability of turbulence through the Equatorial Undercurrent. J. Geophys. Res., 94, 18 003-18 009, doi:10.1029/JC094iC12p18003.

, — - and T. B. Sanford, 1991: Equatorial and off-equatorial fine-scale and large-scale shear variability at $140^{\circ} \mathrm{W}$. J. Geophys. Res., 96, 16913-16 928, doi:10.1029/91JC01317.

,-- , and -1995 : On the parameterization of equatorial turbulence: Effect of fine-scale variations below the range of the diurnal cycle. J. Geophys. Res., 100, 18333-18348, doi:10.1029/ 95JC01513.

Pham, H. T., S. Sarkar, and K. B. Winters, 2012: Near- $N$ oscillations and deep-cycle turbulence in an Upper-Equatorial Undercurrent model. J. Phys. Oceanogr., 42, 2169-2184, doi:10.1175/ JPO-D-11-0233.1.

Philander, S. G. H., 1978: Instabilities of zonal equatorial currents. J. Geophys. Res., 83, 3679-3682, doi:10.1029/JC083iC07p03679.

Proehl, J. A., 1998: The role of meridional flow asymmetry in the dynamics of tropical instability. J. Geophys. Res., 103, $24597-$ 24 618, doi:10.1029/98JC02372.

Qiao, L., and R. H. Weisberg, 1995: Tropical instability wave kinematics: Observation from the Tropical Instability Wave Experiment. J. Geophys. Res., 100, 8677-8693, doi:10.1029/ 95JC00305.

— vations from the Tropical Instability Wave Experiment. J. Phys. Oceanogr., 28, 345-360, doi:10.1175/1520-0485(1998)028<0345: TIWEOF $>2.0 . \mathrm{CO} ; 2$.

Roemmich, D., and J. Gilson, 2009: The 2004-2008 mean and annual cycle of temperature, salinity, and steric height in the global ocean from the Argo program. Prog. Oceanogr., 82, 81-100, doi:10.1016/j.pocean.2009.03.004. 
Smyth, W. D., and J. N. Moum, 2013: Marginal instability and deep cycle turbulence in the eastern equatorial Pacific Ocean. Geophys. Res. Lett., 40, 6181-6185, doi:10.1002/2013GL058403.

,-- , and J. D. Nash, 2011: Narrowband oscillations in the upper equatorial ocean. Part II: Properties of shear instabilities. J. Phys. Oceanogr., 41, 412-428, doi:10.1175/2010JPO4451.1.

,,- L. Li, and S. A. Thorpe, 2013: Diurnal shear instability, the descent of the surface shear layer, and the deep cycle of equatorial turbulence. J. Phys. Oceanogr., 43, 2432-2455, doi:10.1175/JPO-D-13-089.1.

Strutton, P. G., J. P. Ryan, and F. P. Chavez, 2001: Enhanced chlorophyll associated with tropical instability waves in the equatorial Pacific. Geophys. Res. Lett., 28, 2005-2008, doi:10.1029/ 2000 GL012166.

Sun, C., W. D. Smyth, and J. N. Moum, 1998: Dynamic instability of stratified shear flow in the upper equatorial Pacific. J. Geophys. Res., 103, 10323-10337, doi:10.1029/98JC00191.
Sutherland, B. R., 1996: Dynamic excitation of internal gravity waves in the equatorial oceans. J. Phys. Oceanogr., 26, 2398-2419, doi:10.1175/1520-0485(1996)026<2398:DEOIGW>2.0.CO;2.

Thorpe, S. A., 2005: The Turbulent Ocean. Cambridge University Press, $426 \mathrm{pp}$.

Toole, J. M., H. Peters, and M. C. Gregg, 1987: Upper ocean shear and density variability at the equator during TROPIC HEAT. J. Phys. Oceanogr., 17, 1397-1406, doi:10.1175/ 1520-0485(1987)017<1397:UOSADV>2.0.CO;2.

Wijesekera, H., and T. Dillon, 1991: Internal waves and mixing in the upper equatorial Pacific Ocean. J. Geophys. Res., 96, 71157125, doi:10.1029/90JC02727.

Yu, J. Y., and W. T. Liu, 2003: A linear relationship between ENSO intensity and tropical instability wave activity in the eastern Pacific Ocean. Geophys. Res. Lett., 30, 1735, doi:10.1029/2003GL017176.

Yu, Z., J. P. McCreary Jr., and J. A. Proehl, 1995: Meridional asymmetry and energetics of tropical instability waves. J. Phys. Oceanogr., 25, 2997-3007, doi:10.1175/1520-0485(1995)025<2997: MAAEOT $>2.0 . \mathrm{CO} ; 2$. 